\title{
Sensitivity Study of Type-I X-ray Bursts to Nuclear Reaction Rates
}

\author{
Irin Sultana ${ }^{1,3, *}$, Alfredo Estradé ${ }^{1,3}$, Jessica Borowiak $^{1}$, Jacob Elliott ${ }^{1}$, Bradley S. Meyer ${ }^{4}$, \\ and Hendrik Schatz ${ }^{2,3}$ \\ ${ }^{1}$ Department of Physics, Central Michigan University, \\ Mount Pleasant, MI 48859, USA \\ ${ }^{2}$ National Superconducting Cyclotron Laboratory, Michigan State University, \\ East Lansing, MI 48824, USA \\ ${ }^{3}$ JINA Center for the Evolution of the Elements, Michigan State University, \\ East Lansing, MI 48824, USA \\ ${ }^{4}$ Department of Physics and Astronomy, Clemson University, \\ Clemson, SC 29634-0978, USA
}

\begin{abstract}
Type-I X-ray bursts are frequently occurring thermonuclear runaways on the surface of an accreting neutron star. The different nuclear reactions that empower the cataclysmic event play a key role in accurately comparing theoretical models and observations. We investigate the effect of the uncertainties in the nuclear reactions using a ONEZONE model for a set of different compositions and accretion rates that are within the range of the standard observed burst sources. A combination of a full-reaction network and a semi-analytic model is used to obtain the conditions at the time of X-ray burst ignition via simulating the settling process of the accreted material. We then evaluate the sensitivity of the X-ray burst model by varying the proton and alpha-induced reaction rates in JINA REACLIBV2.2 within representative nuclear physics uncertainties.
\end{abstract}

\section{Introduction}

Neutron stars in a low mass X-ray binary accreting hydrogen-rich or helium-rich materials on the surface can frequently produce thermonuclear runaways, known as Type-I X-ray bursts (XRBs) [1] [2] [3]. Type-I X-ray burst occurs every few hours to days with a duration of (10 - 100) s depending on the accretion rate and the accreted composition [4]. Data from different X-ray observatory satellites (e.g. EXOSAT, RXTE, BeppoSAX, and Chandra) play an important role in probing the binary system properties with model-observation comparison [5]. Both observations and models show dependence on the accretion rate and composition of the accreted materials [6].

Type-I X-ray bursts are powered by the triple- $\alpha$ process, the $\alpha p$ process, and the rapid proton capture (rp) process [7] [8]. These nuclear processes include many reactions along the proton-rich side of the nuclear chart. Most of the nuclear reactions are determined theoretically due to the absence of experimental data. To understand the phenomena we need to constraints these nuclear reactions experimentally. Sensitivity studies play an important

\footnotetext{
*e-mail: sulta1c@cmich.edu
} 
role to identify reactions whose reaction rate could be a significant source of uncertainty in the output of the model calculation [4] [9] [10]. These previous studies investigated a limited set of specific models. However, in nature model parameters and the associated burst phenomenology vary widely. Here we use the one-zone model (ONEZONE) [9] to determine the sensitivity of burst observables to nuclear reactions for a broad range of accreted compositions and accretion rates.

\section{Method}

We study the sensitivity of the XRB model to nuclear reaction rates in two steps. First, we obtain the conditions at burst ignition by combining a semi-analytic model (SETTLE code) [11] with a single-zone reaction network code (NucNet Tools) [12]. Second, we use the one-zone X-ray burst model ONEZONE [9] to follow the time-dependent explosive burning during the burst. Reaction rates are varied in ONEZONE to study their impact on the model predictions. We varied each reaction individually within representative nuclear physics uncertainties.

$\mathrm{XRB}$ light curves are dependent on the properties of the binary system along with the nuclear reactions driving the phenomena [9]. Type-I X-ray burst calculations were done for a parameter space based on the observed burst sources [6]. We study different astrophysical conditions with different values of the mass accretion rate and accreted composition. We use the SETTLE code to calculate the temperature and density profile of the surface layers of an accreting neutron star. Figure 1 shows the trajectory obtained from the SETTLE code using an initial accreted hydrogen mass fraction $\mathrm{X}=0.9$, metallicity $\mathrm{Z}=0.02$, where $\mathrm{Z}$ is the mass fraction of metals (species heavier than helium), and $20 \%$ of the Eddington accretion rate $\left(\dot{m}_{E d d}\right)$. The base heating flux from the interior of the star is assumed to be $0.15 \mathrm{MeV} /$ nucleon [11]. The NucNet Tools were then used to obtain the nucleosynthesis along the trajectory of the surface layers. This single-zone network contains isotopes up to xenon and the reaction data from the JINA reaction database REACLIBV2.2 [13]. We adjust the initial hydrogen and helium mass fraction and scale the solar composition for the metals. We use NucNet Tools to follow the evolution of the nuclear composition of the material until the ignition point of an XRB. Calculations are then continued with the ONEZONE model.

The ONEZONE model used for this study contains a network of 713 nuclei in the protonrich side of the nuclear chart from hydrogen to xenon. We varied a set of 1422 proton and alpha-induced reactions in our one-zone model individually including their reverse rates from JINA REACLIBV2.2 [13]. For each calculation, we either increased or decreased one reac-
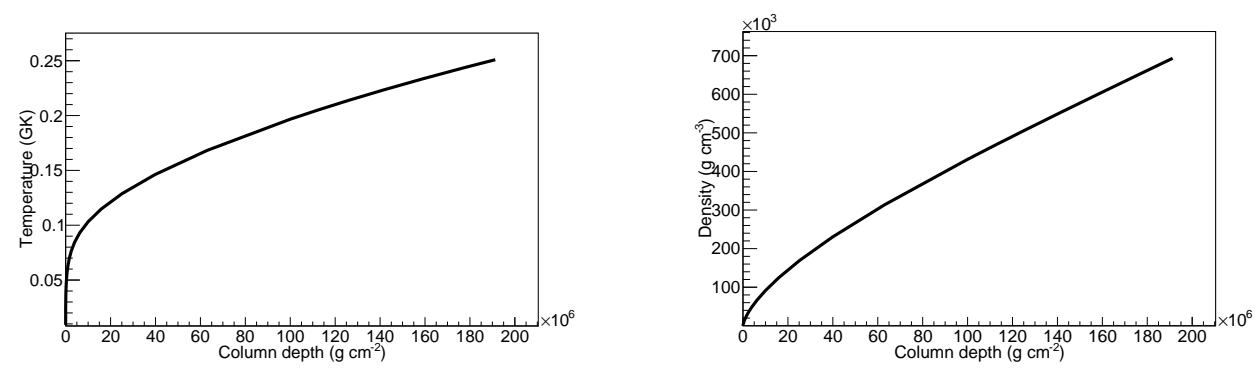

Figure 1. Temperature and density profile of the surface layers for an accreting neutron star using the SETTLE code [11]. See text for initial input. We started our ONEZONE calculation at the column depth of $1.58 \times 10^{8} \mathrm{gcm}^{-2}$. 
tion rate by a factor of 100 . The large variation factor was used to capture all the sensitive reactions [9].

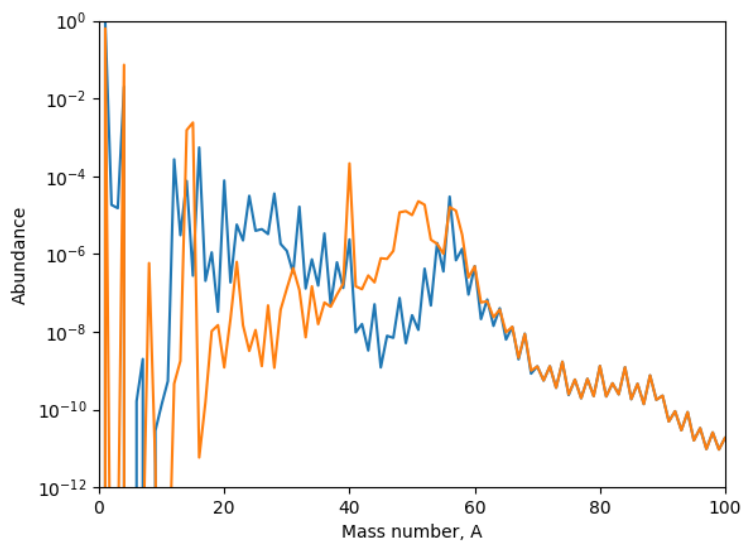

Figure 2. Nuclear composition of the accreted material at the surface (blue line) and at the depth when we change from NucNet Tools to ONEZONE (orange line), after the settling process using the NucNet Tools single-zone reaction network code [12].

\section{Results}

We present sensitivity results for two astrophysical conditions. Both have the same hydrogen mass fraction $X=0.9$ for the accreted material and a mass accretion rate of $0.2 \dot{m}_{E d d}$ while we used two metallicities $\mathrm{Z}=0.02$ and $\mathrm{Z}=0.01$. We refer to the baseline calculation as
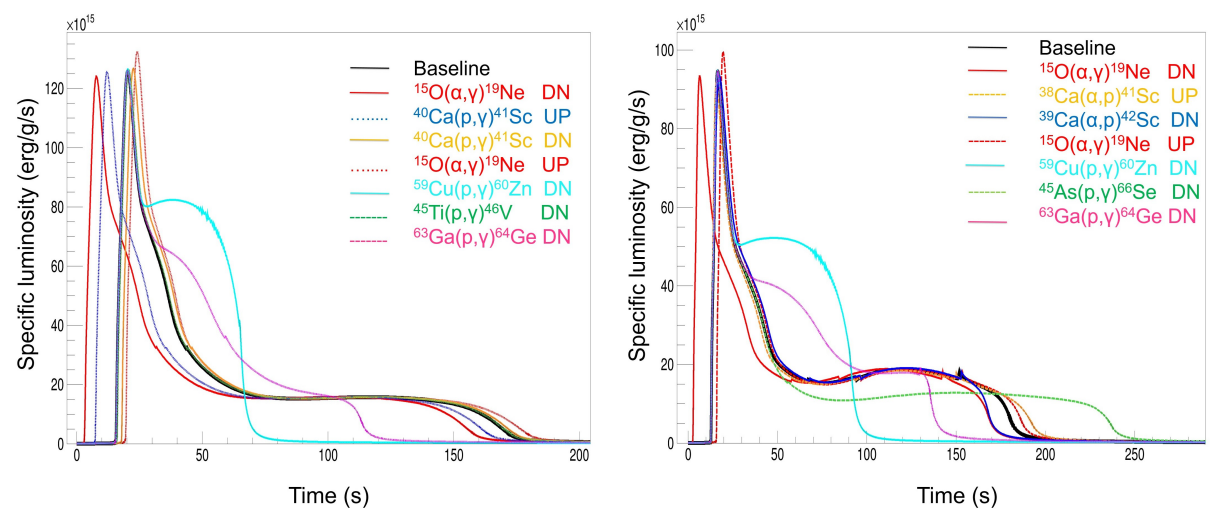

Figure 3. The reactions with higher change in the lightcurve profile as determined through the $F_{s}$ parameter, for models using two different metallicities for the accreted material. For $Z=0.02$ (left), the list of reactions is dominated by the (p, $\gamma)$ reactions while for $\mathrm{Z}=0.01$ (right) the list has higher-ranked $(\alpha, p)$ reactions in comparison. 
the results obtained with unmodified reaction rates of the JINA REACLIBV2.2 library [13] (black line in figure 3). We define the sensitivity parameter $F_{s}$ in the following way,

$$
F_{s}=\left|L_{c}-L_{b}\right|
$$

Here, $L_{b}$ is the maximum value of the specific luminosity for the baseline calculation, and $L_{c}$ is the same value for the case with changed rates. We record the high-impact reactions in table 1. Figure 3 shows the light curves for calculations with high-impact reactions with the largest change from the baseline. For a metallicity of 0.02 , this is mostly dominated by the $(p, \gamma)$ reactions while for 0.01 metallicity there are more high-ranked $(\alpha, p)$ reactions in comparison. In both cases, the highest change in the light curve is produced by the ${ }^{15} \mathrm{O}(\alpha, \gamma){ }^{19} \mathrm{Ne}$ reaction rate.

Table 1. List of high-impact reactions with impact parameter $F_{s}$, for calculations with a hydrogen fraction of 0.9 and 0.2 of Eddington accretion rate, and with two different metallicities $(\mathrm{Z})$ of the accreted material. Calculations, where the reaction rate was increased by a factor of 100 , are labeled as $\mathrm{U}$, and those where the rate was decreased by the same factor are labeled as D.

\begin{tabular}{|c|c|c|c|c|c|c|}
\hline Rank & \multicolumn{2}{|c|}{ Reaction (for $Z=0.02$ ) } & $\mathrm{F}_{s}\left[10^{17} \mathrm{erg} / \mathrm{g} / \mathrm{s}\right]$ & \multicolumn{2}{|c|}{ Reaction(for $\mathrm{Z}=0.01$ ) } & $\mathrm{F}_{s}\left[10^{17} \mathrm{erg} / \mathrm{g} / \mathrm{s}\right]$ \\
\hline 1 & ${ }^{15} \mathrm{O}(\alpha, \gamma){ }^{19} \mathrm{Ne}$ & (D) & 1.18 & ${ }^{15} \mathrm{O}(\alpha, \gamma){ }^{19} \mathrm{Ne}$ & (D) & 0.90 \\
\hline 2 & ${ }^{40} \mathrm{Ca}(p, \gamma){ }^{41} \mathrm{Sc}$ & (U) & 0.95 & ${ }^{15} \mathrm{O}(\alpha, \gamma){ }^{19} \mathrm{Ne}$ & (U) & 0.60 \\
\hline 3 & ${ }^{40} \mathrm{Ca}(p, \gamma)^{41} \mathrm{Sc}$ & (D) & 0.83 & ${ }^{59} \mathrm{Cu}(p, \gamma){ }^{60} \mathrm{Zn}$ & (D) & 0.35 \\
\hline 4 & $\left.{ }^{15} \mathrm{O}(\alpha, \gamma)\right)^{19} \mathrm{Ne}$ & (U) & 0.74 & ${ }^{14} \mathrm{O}(\alpha, p){ }^{17} \mathrm{~F}$ & (U) & 0.26 \\
\hline 5 & ${ }^{59} \mathrm{Cu}(p, \gamma){ }^{60} \mathrm{Zn}$ & (D) & 0.53 & ${ }^{63} \mathrm{Ga}(p, \gamma){ }^{64} \mathrm{Ge}$ & (D) & 0.20 \\
\hline 6 & ${ }^{14} \mathrm{O}(\alpha, p){ }^{17} \mathrm{~F}$ & (U) & 0.34 & ${ }^{19} \mathrm{Ne}(p, \gamma){ }^{20} \mathrm{Na}$ & (D) & 0.18 \\
\hline 7 & ${ }^{44} \mathrm{Ti}(p, \gamma)^{45} \mathrm{~V}$ & (D) & 0.29 & ${ }^{38} \mathrm{Ca}(\alpha, p){ }^{41} \mathrm{Sc}$ & (U) & 0.16 \\
\hline 8 & ${ }^{19} \mathrm{Ne}(p, \gamma){ }^{20} \mathrm{Na}$ & (D) & 0.28 & ${ }^{44} \mathrm{Ti}(p, \gamma){ }^{45} \mathrm{~V}$ & (D) & 0.14 \\
\hline 9 & ${ }^{63} \mathrm{Ga}(p, \gamma){ }^{64} \mathrm{Ge}$ & (D) & 0.27 & ${ }^{65} \mathrm{As}(p, \gamma){ }^{66} \mathrm{Se}$ & (D) & 0.11 \\
\hline 10 & ${ }^{45} \mathrm{Ti}(p, \gamma)^{46} \mathrm{~V}$ & (D) & 0.22 & ${ }^{39} \mathrm{Ca}(\alpha, p){ }^{42} \mathrm{Sc}$ & (D) & 0.10 \\
\hline
\end{tabular}

\section{Discussions}

The different properties of a binary system with an accreting neutron star can lead to X-ray bursts with significantly different characteristics. For metallicity $\mathrm{Z}=0.02$, the conditions at the burst ignition were a hydrogen mass fraction $\mathrm{X}=0.63$, helium mass fraction $\mathrm{Y}=$ 0.29 , temperature $\mathrm{T}=0.26 \mathrm{GK}$, and pressure $\mathrm{P}=5.05 \times 10^{22} \mathrm{ergcm}^{-3}$. For metallicity $\mathrm{Z}=$ 0.01 , ignition conditions were, hydrogen mass fraction $\mathrm{X}=0.65$, helium mass fraction $\mathrm{Y}$ $=0.28$, temperature $\mathrm{T}=0.25 \mathrm{GK}$, and pressure $\mathrm{P}=7.19 \times 10^{22} \mathrm{ergcm}^{-3}$. Our results show that depending on the different ignition conditions different nuclear processes have a stronger influence on the burst light curve.

We have developed a sensitivity study that considers the condition of burst ignition for a range of system parameters. Our preliminary results show the features of the accreted material have a strong impact on the type of important reactions.

\section{Acknowledgements}

We would like to thank Zach Meisel for useful discussions. This work was supported by U.S. DOE award DE-SC0020406 and by NSF award PHY-1430152 and PHY-1913554. 


\section{References}

[1] H. Schatz, A. Aprahamian, J. Görres, M. Wiescher, T. Rauscher, J. Rembges, F. Thielemann et al., Physics Reports 294, 167 (1998)

[2] A. Parikh, J. José, G. Sala, C. Iliadis, Progress in Particle and Nuclear Physics 69, 225 (2013), 1211.5900

[3] Z. Meisel, A. Deibel, L. Keek, P. Shternin, J. Elfritz, Journal of Physics G Nuclear Physics 45, 093001 (2018), 1807.01150

[4] A. Parikh, J. José, F. Moreno, C. Iliadis, 52, 409 (2008), 0806. 2975

[5] A. Heger, A. Cumming, D.K. Galloway, S.E. Woosley, 671, L141 (2007), 0711. 1195

[6] D.K. Galloway, A.J. Goodwin, L. Keek, 34, e019 (2017), 1703.07485

[7] R.K. Wallace, S.E. Woosley, 45, 389 (1981)

[8] H. Schatz, A. Aprahamian, V. Barnard, L. Bildsten, A. Cumming, M. Ouellette, T. Rauscher, F.K. Thielemann, M. Wiescher, 688, 150 (2001), nucl-th/0201033

[9] R. Cyburt, A. Amthor, A. Heger, E. Johnson, L. Keek, Z. Meisel, H. Schatz, K. Smith, The Astrophysical Journal 830, 55 (2016)

[10] H. Schatz, W.J. Ong, 844, 139 (2017), 1610.07596

[11] A. Cumming, L. Bildsten, The Astrophysical Journal 544, 453 (2000)

[12] B.S. Meyer, Proceedings of Science NIC XII, 096 (2013)

[13] R.H. Cyburt, A.M. Amthor, R. Ferguson, Z. Meisel, K. Smith, S. Warren, A. Heger, R.D. Hoffman, T. Rauscher, A. Sakharuk et al., 189, 240 (2010) 